\title{
Fully Enclosed Microfluidic Paper-Based Analytical Devices
}

\author{
Kevin M. Schilling, Anna L. Lepore, Jason A. Kurian, and Andres W. Martinez* \\ Department of Chemistry and Biochemistry, California Polytechnic State University, San Luis Obispo, California 93407, United \\ States
}

Supporting Information

ABSTRACT: This article introduces fully enclosed microfluidic paper-based analytical devices (microPADs) fabricated by printing toner on the top and bottom of the devices using a laser printer. Enclosing paper-based microfluidic channels protects the channels from contamination, contains and protects reagents stored on the device, contains fluids within the channels so that microPADs can be handled and operated more easily, and reduces evaporation of solutions from the channels. These benefits extend the capabilities of microPADs for applications as low-cost point-of-care diagnostic devices.

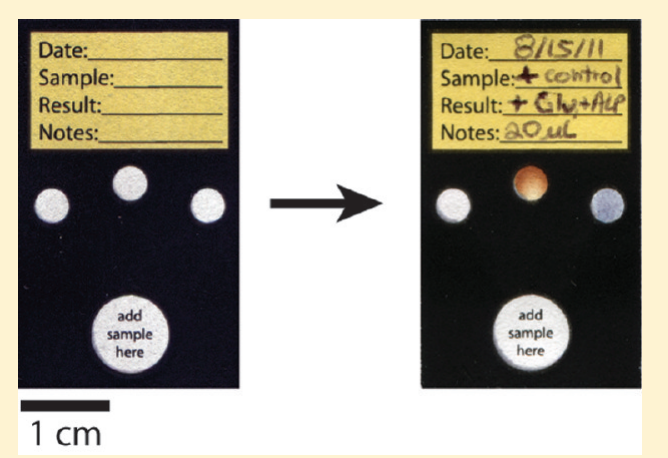

$\mathrm{T}$ his article presents a simple method for sealing the top and bottom faces of channels in microfluidic paper-based analytical devices (microPADs) to make fully enclosed microPADs. The devices were enclosed with an impermeable layer of toner printed on the top and bottom of the device using a laser printer (Figure 1). Fully enclosed microPADs have several advantages over conventional microPADs in which the top and bottom faces of the channels are open and exposed to the environment. ${ }^{1}$ We demonstrated that enclosing microPADs reduces evaporation of fluids from the channels, which results in faster wicking rates compared to conventional open channels, that the reagents for assays can be applied before sealing the devices and stored on-chip, and that a fully enclosed microPAD can be used to detect clinically relevant concentrations of glucose and alkaline phosphatase (ALP).

Microfluidic devices made out of paper have been shown recently to have many characteristics that make them wellsuited for use as point-of-care diagnostic devices for detection of disease and other analytes in remote settings; key among these characteristics are low cost, ease of operation, and ability to function without any external power supplies or supporting equipment. $^{2}$ In microPADs, fluids wick along channels patterned into sheets of paper or other porous membranes such as nitrocellulose. ${ }^{2}$ Due to the structural characteristics of paper, the top and bottom faces of the channels in microPADs typically remain open and exposed to the environment. This common feature of microPADs could be considered a limitation when compared to conventional microfluidic devices, where fluids are fully enclosed within channels etched or molded into glass or plastic. MicroPADs with open channels have three principal limitations: (i) the channels are constantly at risk of being contaminated during fabrication, transportation, or operation; (ii) when the channels wick fluids, a large amount of the fluid is lost due to evaporation, which can limit the distance that the fluid can wick along a channel and significantly increase the time required for the fluid to wick a given distance; (iii) when a fluid is added to a device, the device must be suspended in air, otherwise the fluid will wick onto any surface in contact with the device, which can result in contamination and loss of samples.

Several attempts have been made to seal one or both faces of microPADs to overcome the problems of open channels. Fenton et al. used polyester-backed nitrocellulose to make microPADs so that the back of the devices would be sealed with an impermeable layer of polyester. ${ }^{3}$ In the same article, tape was used to seal the top of some devices resulting in the first examples of fully enclosed microPADs. Double-sided tape was used to enclose paper-based channels in three-dimensional microPADs and bond the layers of paper together in the device. ${ }^{4}$ Our experience with using tape to enclose microPADs is that it forms an effective seal with the device when the channels are dry, but adhesion is poor when the channels are wet. Furthermore, if inlet and outlet holes are required in the tape, holes must be cut into the tape, and the tape must be aligned with the microPAD. A third disadvantage of using tape to enclose microPADs is that the adhesives in some tapes can diffuse into paper over time, making the paper less hydrophilic. Another method of sealing one face of microPADs was introduced by Olkkonen et al. and involved flexographically printing a thin layer of polystyrene on one side of the paper. ${ }^{5}$ This method cannot be used to fully enclose a device because the paper becomes hydrophobic when both the top and bottom of the device are sealed. An alternative approach to overcoming the problems of open channels involves placing microPADs inside a plastic case such as a Petri dish. ${ }^{6}$ The case protects the

Received: October 27, 2011

Accepted: December 16, 2011

Published: January 9, 2012 


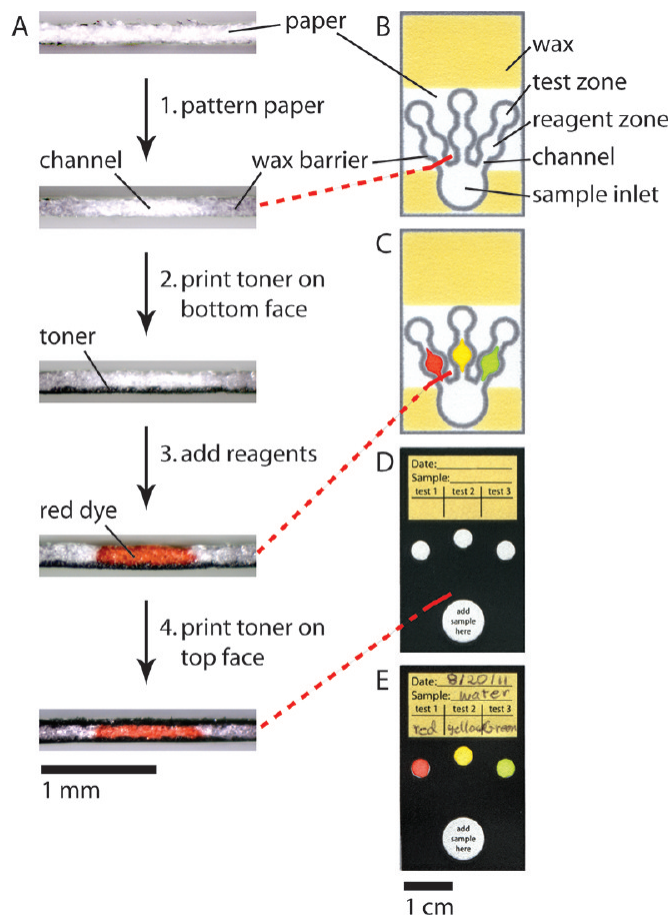

Figure 1. Fabrication of fully enclosed microPADs. (A) Cross sections of microPADs during the four steps required to fabricate fully enclosed channels. The channels in the device were enclosed by wax barriers on the left and right and by toner on the top and bottom. (B) A device after it was patterned by wax printing. The device has a single sample inlet that leads to three test zones via reagent-storage zones. (C) The same device shown in panel B after adding $1 \mu \mathrm{L}$ of aqueous solutions of red, yellow, and green dyes to the reagent-storage zone. (D) A fully enclosed microPAD after sealing the top of the device shown in panel $\mathrm{C}$ with toner. (E) The same device shown in panel $\mathrm{D}$ after adding 20 $\mu \mathrm{L}$ of water to the sample inlet. The water wicked through the reagentstorage zones where it dissolved the dyes and carried them into the test zones. The red dashed lines indicate the locations where the cross sections shown in panel A were taken.

device and limits evaporation, but it also adds to the complexity and cost of the device.

Sealing the top and bottom faces of paper-based channels with toner provides a simple way of enclosing microPADs using equipment that is already available in most offices and laboratories. Laser printers and toner have been used extensively in the fabrication of conventional microfluidic devices. ${ }^{7}$ More recently, toner was used to pattern the surface of paper to make patterned hydrogels. ${ }^{8,9}$ When toner is printed on microPADs, it creates a thin layer of plastic that is thermally bonded to the device and will not diffuse into the channels or come off when the channels are wet. The layer of toner can also be printed on microPADs in any pattern or color, so inlet and outlet holes, color charts, operating instructions, or labels can be added easily to the fully enclosed devices. This method of fabrication should bring greater capabilities to microPADs and extend the applications of this class of devices.

\section{EXPERIMENTAL DESIGN}

In developing fully enclosed microPADs, we had four principle objectives: (i) develop and optimize a procedure for sealing the top and bottom faces of paper-based devices with toner; (ii) demonstrate that the wicking properties of fully enclosed channels are not adversely affected by the layer of toner; (iii) demonstrate that the method of fabrication is compatible with diagnostic assays and, specifically, that reagents for an assay can be sealed within the layers of toner; (iv) demonstrate a prototype of a fully enclosed paper-based diagnostic device.

To develop a method for sealing the devices, we first had to determine the number of layers of toner required to form an impermeable membrane on the paper. We noted in initial experiments that a single layer of toner printed on paper had many defects including holes and cracks through which aqueous solutions passed easily. We also found that printing several layers of toner on top of each other produced thicker layers of toner with less frequent defects, so we designed an experiment to determine the number of layers of toner on the surface of paper required to form a barrier that had no defects and therefore was impermeable to water.

The ability of microPADs to wick fluids by capillary action is the most distinctive characteristic of this class of devices, and we wanted to make sure that the wicking properties of fully enclosed channels were not adversely affected by the toner. We studied the wicking properties of enclosed channels and compared these to the wicking properties of open channels. Yellow toner was used to enclose the channels for these experiments so that the results could be visualized through the toner.

One advantage of enclosing microPADs with toner is that reagents on the devices can also be enclosed and protected from contamination and exposure to light. In order to enclose reagents, they must be added to a microPAD before the top layer of toner is printed on the device. This limitation is problematic because, as part of the laser printing process, the paper is passed through a fuser heated to $\sim 180^{\circ} \mathrm{C}$, ${ }^{10}$ and any thermally sensitive reagents may not be compatible with this fabrication method. To determine the effect of the printing process on thermally sensitive reagents, we studied a colorimetric glucose assay. We chose this assay because it is an enzymatic assay that is quantitative and has been used extensively with microPADs. ${ }^{11}$ By comparing the results of assays from devices enclosed with toner to results from devices with no toner, we determined the effect of the printing process on the reagents. Since the glucose assay is an enzymatic assay, the results of these experiments should be relevant to other assays that rely on proteins.

All of the devices were fabricated with Whatman no. $1 \mathrm{Chr}$ chromatography paper because it is made of pure cellulose, wicks fluids uniformly, and has become a standard starting material for fabricating microPADs. We patterned the channels in the paper by wax printing because this method is simple, rapid, and only requires a solid-ink printer and an oven. ${ }^{12}$ Several other methods for patterning paper have been described in the literature and should be compatible with this method of enclosing microPADs. ${ }^{2,5,13,14}$ Cutting channels out of paper is another method of fabricating microPADs. ${ }^{3}$ Although it would probably not be possible to print toner on devices made by cutting, it should be possible to print toner on a sheet of paper before cutting out the devices.

\section{EXPERIMENTAL DETAILS}

Determining the Amount of Toner Required To Form an Impermeable Barrier on Paper. Rectangular pieces of paper $(6 \mathrm{~cm} \times 5 \mathrm{~cm})$ sealed on both the front and back with one, two, three, four, or five layers of toner were placed between two pieces of blotting paper $(5 \mathrm{~cm} \times 5 \mathrm{~cm})$, and the three pieces of paper were pressed together by placing a piece 
of glass $(10 \mathrm{~cm} \times 15 \mathrm{~cm}, 74 \mathrm{~g})$ on top of them in such a way that a single $1 \mathrm{~cm}$ wide edge of the paper sealed with toner was exposed. The exposed edge of the sealed paper was dipped into an aqueous blue solution ( $1 \mathrm{mM}$ erioglaucine) until the paper had wicked enough solution to become fully saturated $(\sim 15$ $\mathrm{min}$ ), and the assembly was allowed to sit for an additional 15 $\mathrm{min}$. The pieces of blotting paper were then removed from the assembly, and any blue spots on them were counted. Each spot on the blotting paper was attributed to an individual leak in the layer of toner.

Fabricating Fully Enclosed MicroPADs. Fully enclosed microPADs were fabricated in four steps as shown in Figure 1. Whatman no. $1 \mathrm{Chr}$ chromatography paper was patterned by wax printing. ${ }^{12}$ Solid ink (wax) was printed on paper using a Xerox Phaser 8560 printer set to photo quality. The paper was then heated for $15 \mathrm{~min}$ in a convection oven set to $138{ }^{\circ} \mathrm{C}$ to melt the solid ink. Under these conditions, a $200 \mu \mathrm{m}$ wide printed line resulted in a $1 \mathrm{~mm}$ wide wax barrier. The devices were cooled to room temperature for $5 \mathrm{~min}$, and then the top of the devices were coated with four layers of toner using a Samsung CLP-620ND printer set to best quality. The devices were allowed to equilibrate with ambient humidity for at least 5 min between each print cycle. Reagents were added to the devices and dried under ambient conditions. Finally, four layers of toner were printed on the back of the devices, again allowing the devices to sit for $5 \mathrm{~min}$ between each print cycle.

Evaluating the Wicking Properties of Fully Enclosed Channels. Devices were prepared with three straight channels ( $8 \mathrm{~cm}$ long and $2 \mathrm{~mm}$ wide) enclosed with zero, four, and six layers of yellow toner (Figure 2A). The devices were dipped vertically to a depth of $2 \mathrm{~mm}$ into a fluid reservoir containing aqueous blue dye, and the fluid fronts were tracked over time as fluid wicked up the channels at an ambient relative humidity of $53 \%$. The experiment was repeated inside a glass chamber to simulate $100 \%$ relative humidity.

Enclosing Reagents in MicroPADs. A microPAD with a sample inlet, a reagent-storage zone, and a test zone was prepared (Figure 3). An amount of $1 \mu \mathrm{L}$ of a reagent solution containing $0.6 \mathrm{mM}$ potassium iodide, $0.3 \mathrm{mM}$ trehalose, 67000 $\mathrm{U} / \mathrm{L}$ glucose oxidase, and $67000 \mathrm{U} / \mathrm{L}$ horseradish peroxidase in $1 \times$ PBS (phosphate-buffered saline, $\mathrm{pH} 7.4$ ) was spotted in the reagent-storage zone, dried under ambient conditions for 5 min, and enclosed with four layers of toner. Fifteen microliters of $200 \mathrm{mM}$ glucose in $1 \times$ PBS was added to the sample inlet. The solution wicked across the reagent-storage zone, where it dissolved the reagents and transported them into the test zone, where the color from the assay developed. After $1 \mathrm{~h}$, the devices were scanned using an Epson Perfection V700 scanner, and the intensity of the color in the test zones was measured using Adobe Photoshop. ${ }^{11}$

The activity of the glucose oxidase and horseradish peroxidase was determined from the intensity of the color produced by the assay. A calibration curve for the intensity of the color in the test zone versus the concentrations of glucose oxidase and horseradish peroxidase was prepared using open devices with no toner (Supporting Information Figure S-1). The concentrations of glucose oxidase and horseradish peroxidase were varied from $0 \mathrm{U} / \mathrm{L}$ or $0 \%$ enzyme to 67000 $\mathrm{U} / \mathrm{L}$ or $100 \%$ enzyme. The reagent-storage zones of devices with $100 \%$ enzyme were then enclosed with four layers of toner. The glucose test was performed using the enclosed devices, and the results were compared to the calibration curve

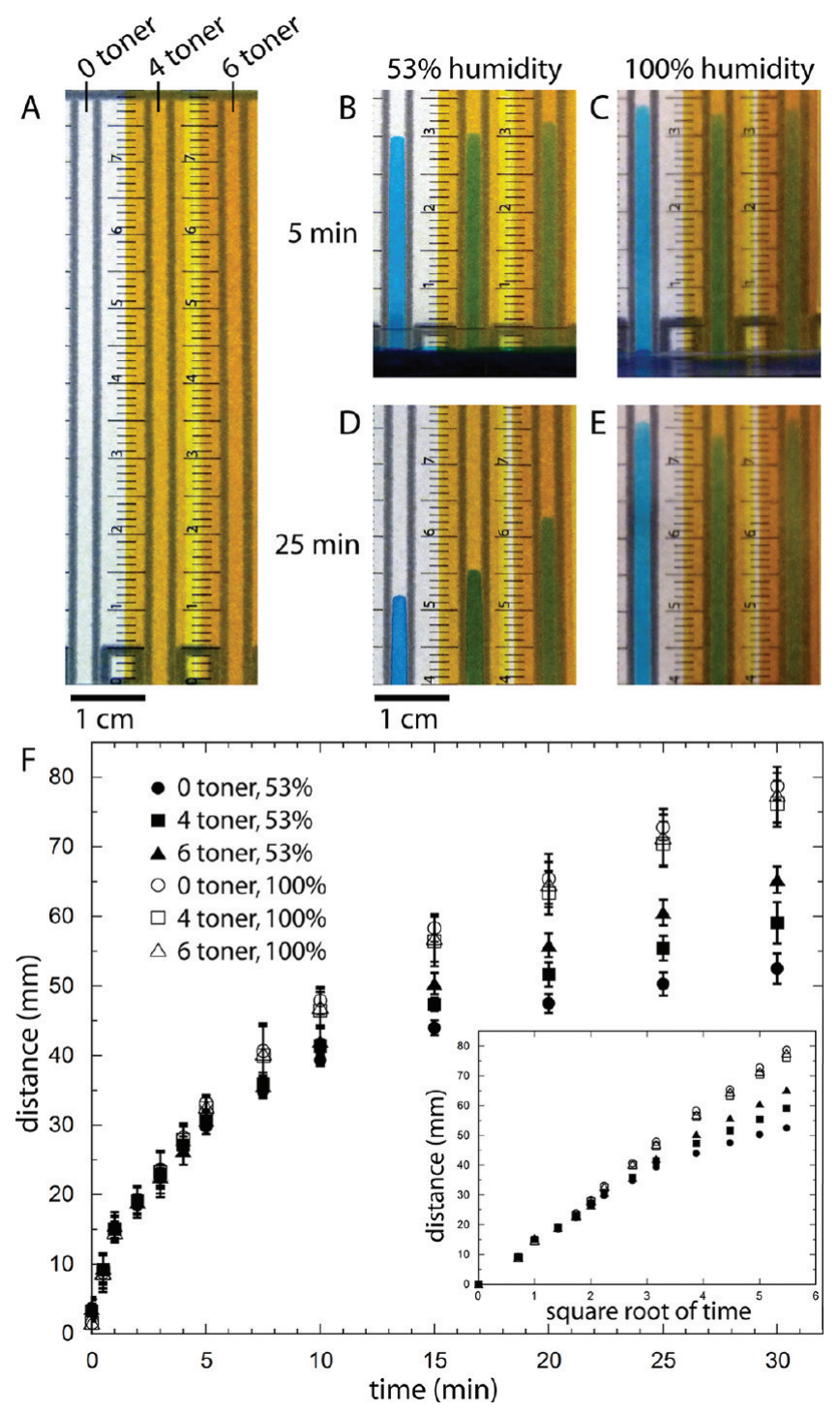

Figure 2. Capillary wicking in fully enclosed channels. (A) The device used for the wicking experiments. The three channels were $8 \mathrm{~cm}$ in length and $2 \mathrm{~mm}$ in width. The channels were enclosed with zero $(0$ toner), four (4 toner), or six (6 toner) layers of toner, respectively. (B-E) Images of the results from wicking experiments 5 and $25 \mathrm{~min}$ after dipping the bottom of the device into a reservoir of aqueous blue dye. The experiments were carried out at $53 \%$ and $100 \%$ relative humidity. Up to $5 \mathrm{~min}$ after starting the experiment, all channels had virtually identical results. After $25 \mathrm{~min}$, large differences in the distance that each fluid wicked were evident. (F) Results of the wicking experiments plotted as distance vs time. Each point on the graph represents the average of six experiments. The error bars represent the 95\% confidence interval. The inset shows the distance plotted vs the square root of time to illustrate that the experiments at $100 \%$ relative humidity follow the Washburn equation.

to calculate the activity of the enzymes after the printing process.

Preparing a Fully Enclosed MicroPAD. MicroPADs with a single sample inlet leading to three test zones via three reagent-storage zones were fabricated (Figure 4). Reagents for the glucose assay were enclosed in the central reagent-storage zone. Reagents for an alkaline phosphatase (ALP) assayBCIP/NBT 1-C AP substrate from VWR-were added to the test zone on the right side of the device. The test zone on the left side of the device was left as a control. The devices were 


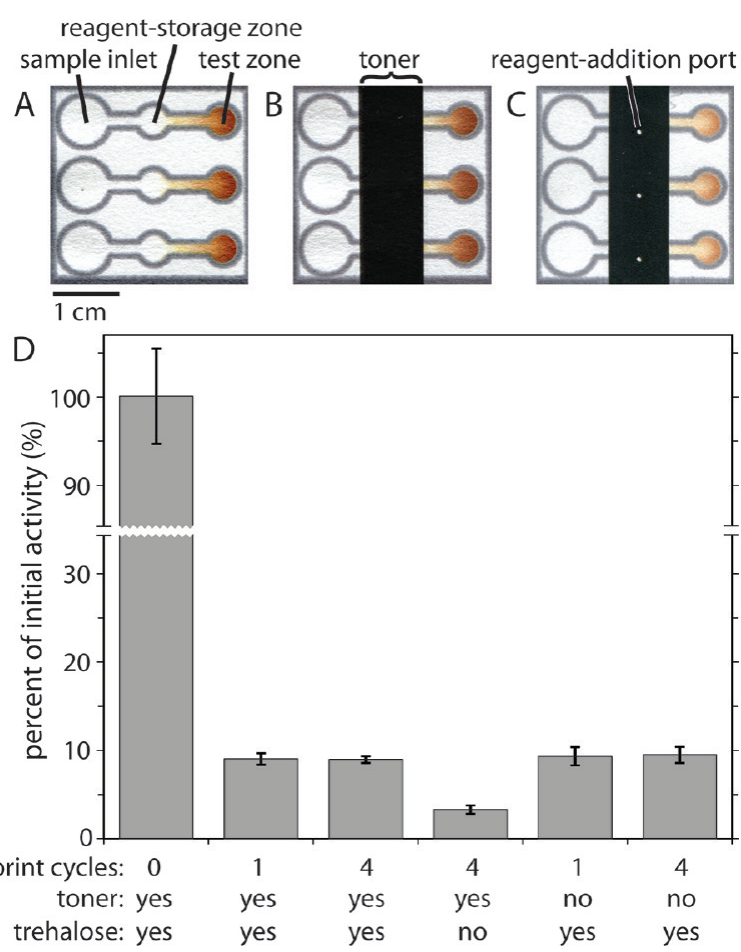

Figure 3. Enclosing reagents with toner. (A) The device used to measure the effect of the printing process on the reagents for the glucose assay. The reagents were spotted and dried in the reagentstorage zone before being enclosed with toner. (B) A device with four layers of toner over the reagent-storage zones. (C) A device with reagent-addition ports. The reagents were added to the device through the reagent-addition ports after fabricating the device. Images shown in panels A-C were taken after running the assays with $200 \mathrm{mM}$ glucose. (D) Results from the enzyme survival experiments. Enzymes added through the reagent-addition port (print cycles $=0$ ) retained $100 \%$ of their initial activity. When the enzymes pass through the printer (print cycles $=1$ or 4 ), the activity of the enzymes was reduced to $10 \%$ of the initial activity regardless of whether toner is printed over the reagents or not. Without trehalose, the activity of the enzymes was reduced to $3 \%$ of the initial activity. Each bar represents the average of eight experiments, and the error bars represent the $95 \%$ confidence interval.

tested using a solution containing $10 \mathrm{mM}$ glucose and $500 \mathrm{U} / \mathrm{L}$ ALP in $1 \times$ PBS.

\section{RESULTS AND DISCUSSION}

Four Layers of Toner Are Required To Seal MicroPADs. Four layers of toner formed a virtually impermeable barrier on paper (Table 1). We estimate that the total surface area of the channels in a typical paper-based microfluidic device is $1 \mathrm{~cm}^{2}$. On the basis of this assumption and the results shown in Table 1, we calculated the probability that a typical device would have no leaks by modeling the occurrence of leaks as a spatial Poisson process. ${ }^{15}$ The probability $P$ of $k$ leaks occurring in a surface area $A$ is given by

$$
P(k \text { leaks in } A)=\mathrm{e}^{-\lambda A}(\lambda A)^{k} / k \text { ! }
$$

where $\lambda$ is the average number of leaks per unit area. Devices sealed with three layers of toner have an estimated probability of 0.0025 of having no leaks, which means virtually all devices with three layers of toner or fewer would be expected to leak at some point along the channel. Devices sealed with four layers of toner have an estimated probability of 0.99 of having no leaks, so we would expect that, on average, 99 out of every 100
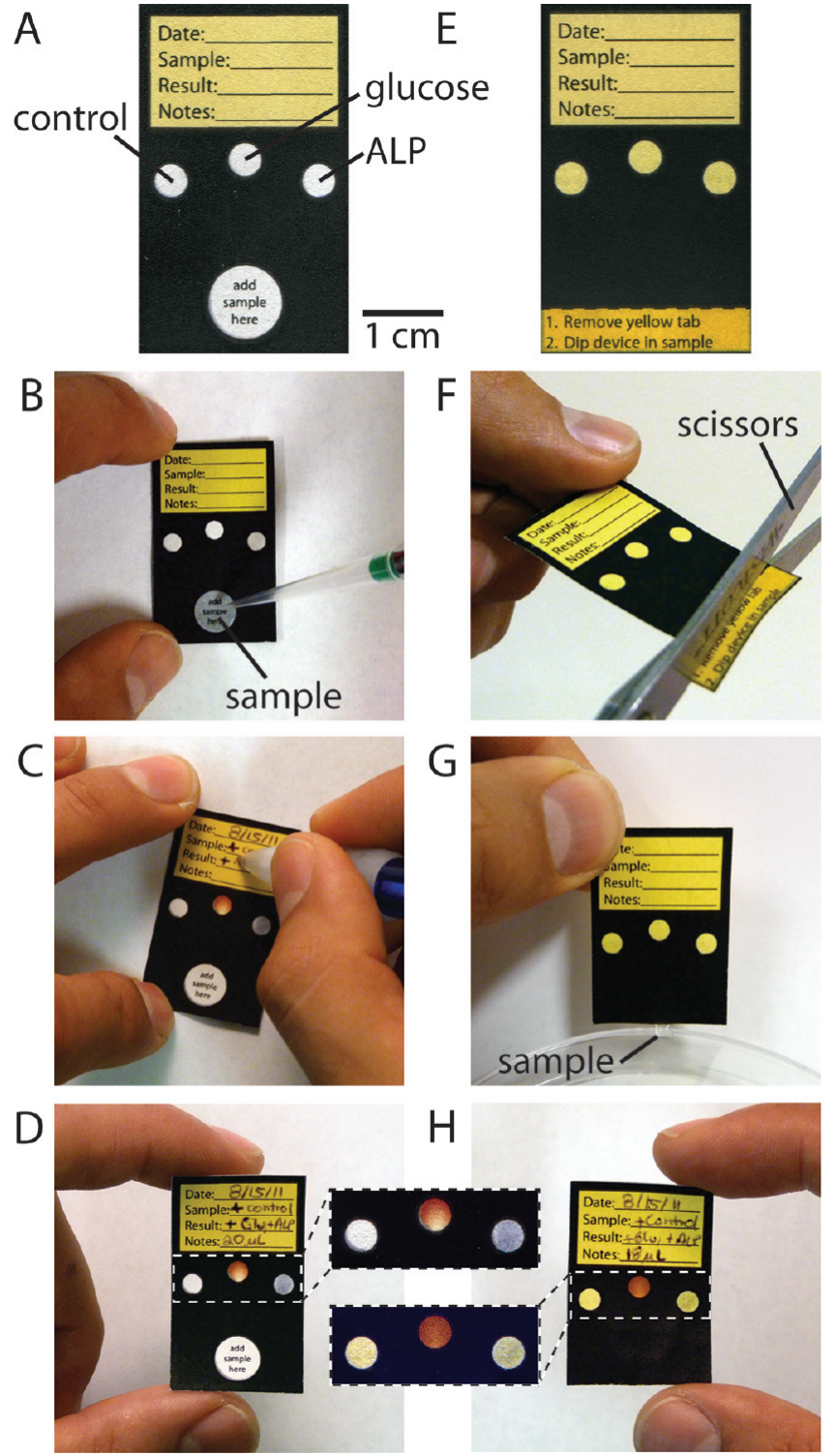

Figure 4. Fully enclosed microPADs for detecting glucose and alkaline phosphatase (ALP). The channels within the device are identical to those shown in Figure 1B. (A) A fully enclosed microPAD with a sample inlet and holes over the test zones. (B) A $20 \mu \mathrm{L}$ sample containing $10 \mathrm{mM}$ glucose and $500 \mathrm{U} / \mathrm{L}$ ALP is added to the device. (C) The device is labeled with a pen. (D) The device displaying the results of the assays. (E) A fully enclosed microPAD with yellow toner enclosing the test zones and sample inlet. (F) The bottom of the device is cut off with scissors to expose the sample inlet. (G) The bottom of the device is dipped into a drop of sample. $(\mathrm{H})$ The device displaying the results of the assays.

microPADs sealed with four layers of toner would be fully enclosed with no leaks. Five layers of toner provide slightly better results than four layers of toner, but we concluded that the benefit of printing an additional layer of toner did not outweigh the cost in terms of supplies and time required to print the additional layer of toner.

We also explored the possibility of enclosing microPADs with solid ink from the Xerox Phaser printer instead of toner. Printing one layer of solid ink at the highest resolution provided results that were comparable to two layers of toner. We found that it was not possible to print multiple layers of solid ink on top of each other because the ink would not adhere 
Table 1. Determining the Number of Layers of Toner Required To Form an Impermeable Layer on Paper ${ }^{a}$

\begin{tabular}{|c|c|c|c|c|c|}
\hline $\begin{array}{l}\text { toner } \\
\text { layers }\end{array}$ & $\begin{array}{c}\text { area sampled } \\
\left(\mathrm{cm}^{2}\right)\end{array}$ & \# of leaks & leaks $/ \mathrm{cm}^{2}$ & image & $\begin{array}{l}\mathrm{P}(0 \text { leaks } \\
\left.\text { in } 1 \mathrm{~cm}^{2}\right)\end{array}$ \\
\hline 1 & 5 & 240 & 48 & $\therefore 8$ & $1.4 \times 10^{-21}$ \\
\hline 2 & 5 & 60 & 12 & $\therefore \therefore$ & $6.1 \times 10^{-6}$ \\
\hline 3 & 5 & 30 & 6 & - & 0.0025 \\
\hline 4 & 156 & 2 & 0.013 & - & 0.99 \\
\hline 5 & 156 & 0 & 0 & & 1.0 \\
\hline $\begin{array}{c}1 \\
\text { (solid ink) }\end{array}$ & 5 & 110 & 22 & $\begin{array}{l}1 \\
1\end{array}$ & $2.0 \times 10^{-9}$ \\
\hline
\end{tabular}

${ }^{a}$ The image shows a $1 \mathrm{~cm}^{2}$ sample of the blotting paper after the experiment was complete. The probability $P$ that a $1 \mathrm{~cm}^{2}$ area of paper would have no leaks was calculated by modeling the occurrence of leaks as a spatial Poisson process.

to itself. For this reason, we concluded that solid ink was not a viable material for enclosing microPADs and that toner was better suited for this work.

The four colors of toner-red, blue, yellow, or black-all gave the same result: four layers of toner were required to form a virtually impermeable barrier on microPADs. This result is important because printing light-colored toner allows for visualization of the channels through the toner barrier. Yellow toner was the most translucent of the four colors of toner and was used for all experiments where we needed to track the movement of fluids in the channels. Black toner was used for all the other devices because black toner is less expensive than colored toners. The cost of black toner required to enclose microPADs was calculated to be $\$ 0.01 \mathrm{~cm}^{-2}$ of device.

When printing consecutive layers of toner on the same piece of paper, we observed that the toner coverage decreased with each consecutive print cycle (Supporting Information Figure S2 ). We eventually traced the problem to the paper not having enough time to recover from one print cycle to the next. Low humidity has been shown to negatively affect toner adhesion, ${ }^{16}$ and we believe that after the first print cycle, the moisture content of the paper is reduced significantly from passing through the fuser. We found that waiting $5 \mathrm{~min}$ between each print cycle was sufficient time to allow the paper to reequilibrate with the ambient humidity so that the toner coverage would not be compromised.

It is likely that different laser printers will yield different results depending on the amount of toner that the printer deposits on the surface of the paper. It may also be possible to develop a specialized printer that could enclose microPADs in a single print cycle. This technology would simplify the fabrication of fully enclosed microPADs significantly and would be highly desirable for large-scale production of fully enclosed devices. We did not attempt to modify our printer because our intention was to develop a method for enclosing microPADs that would be readily accessible to all scientists and rely only on commercially available equipment and materials.

Toner Improves the Wicking Rate in MicroPADs. Enclosing channels with toner increases the wicking rate of aqueous solutions in the channel compared to conventional open channels by reducing the amount of evaporation from the channel (Figure 2). Channels enclosed with six layers of toner had the highest wicking rates of the three channels tested, which suggests that the evaporation from the channel can be decreased even further by enclosing it with additional layers of toner. We found that the wicking rate reached a maximum when eight layers of toner were printed on each face of the paper, but we did not study channels with more than six layers of toner in detail because the differences in the wicking rates were small and inconsistent.

To demonstrate that the differences in wicking rates were due to evaporation and not some other effect, the wicking experiments were also conducted inside a glass chamber containing a large fluid reservoir in order to simulate an environment with $100 \%$ relative humidity and minimize evaporation from the channels. As expected, inside the chamber, the wicking rates of the three channels were indistinguishable (Figure 2F). We also observed that the wicking rates for all three channels were much higher in the humidity chamber than at ambient humidity, which suggests that some amount of evaporation is still occurring from the channels enclosed with toner.

Plotting the distance traveled by the fluid front versus the square root of time yields a straight line for all the experiments carried out at high humidity (Figure $2 \mathrm{~F}$ ). This result is predicted by the Washburn equation:

$$
L=(\gamma r t \cos \theta / 2 \eta)^{1 / 2}
$$

where $L$ is the distance penetrated by a liquid flowing under capillary pressure into a capillary in time $t, \gamma$ is the surface tension, $r$ is the average radius of the capillary, $\theta$ is the contact angle, and $\eta$ is the viscosity of the liquid. ${ }^{17}$ Since the viscosity, surface tension, contact angle, and capillary radius are all constant in our experiments, the equation predicts that the distance traveled by the fluid front should be directly proportional to the square root of time. The agreement between the theory and our experimental measurements supports the conclusion that the presence of the toner on the paper has no effect on the wicking properties of the paper and that the differences observed in the wicking rates at low humidity between open and fully enclosed channels can be attributed to evaporation from the channels.

The wicking rates of all the channels at low and high humidity were indistinguishable during the first $5 \mathrm{~min}$ or $\sim 3 \mathrm{~cm}$ of the channels (Figures $2 \mathrm{~B}$ and $\mathrm{C}$ ). This result suggests that the wicking rates of short channels $(<3 \mathrm{~cm})$ are not significantly affected by the ambient relative humidity. For devices with long channels, the differences in wicking rates between enclosed and open channels can have significant effects on the times required to operate the device. For example, at $53 \%$ relative humidity, a $2 \mathrm{~mm}$ wide open channel would require $25 \mathrm{~min}$ to wick fluid a distance of $5 \mathrm{~cm}$. The same channel would only require $17 \mathrm{~min}$ to wick the fluid if enclosed with four layers of toner and 15 
min if enclosed with six layers of toner. These differences would be even greater at lower humidity or if narrower channels were used. It may also be possible to control the wicking rates of channels by varying the amount of toner used to enclose them.

Reagents Can Be Enclosed in MicroPADs. The activity of the glucose oxidase and horseradish peroxidase was reduced to $\sim 10 \%$ of the initial activity after the devices were enclosed with toner (Figure 3). This result suggests $90 \%$ of the enzyme added to the device was denatured during the printing process. Although it is not desirable to lose $90 \%$ of a reagent, we feel that the method is still viable for preparing devices because only a small amount of reagent is used on each device and, even with only $10 \%$ of the initial activity of the enzymes, it is still possible to measure clinically relevant concentrations of glucose as low as $1 \mathrm{mM}$. We observed identical results when the devices were run through the printer with no toner printed over the reagents. This experiment confirmed that it was the printing process and not the toner that reduced the activity of the enzymes. We also confirmed independently that the potassium iodide and trehalose were not affected by the printing process.

As an alternative to enclosing reagents by adding them to microPADs before printing the top layer of toner, we developed a reagent-addition port-a $1 \mathrm{~mm}$ diameter hole in the layer of toner directly over the reagent-storage zone-so that reagents could be added to the device after fabrication was complete (Figure 3C). Enzymes for the glucose assay added through the reagent addition port retained $100 \%$ of their initial activity (Figure 3D). The reagent-addition port has the advantage that no reagent is lost or denatured during the printing process, and the reagents are still protected by the layer of toner except for the small area under the port. Since reagents are applied to the microPAD after it is fabricated, a common device could be used for many different assays, and the reagents could even be applied at the point of care.

Although we expected the printing process would reduce the activity of the enzymes for the glucose assay, an unexpected result was that the effect was independent of the number of print cycles. The activity of the enzymes was always reduced to $10 \%$ of the initial activity, regardless of whether the device had gone through the printer one, two, three, or four times. One possible explanation for this observation is that the enzymes that survived the printing process were located in the center of the paper where they were better protected from the high temperatures of the fuser.

We added trehalose to the reagents for the glucose assay because trehalose has been shown to increase the stability of proteins on microPADs. ${ }^{11}$ When we enclosed reagents for the glucose assay without adding trehalose to the reagent mixture, only $3 \%$ of the initial activity of the enzymes was observed. This result confirms that trehalose stabilizes the enzymes and suggests that this experiment may provide a simple way of identifying enzyme stabilizing agents.

Fully Enclosed Paper-Based Diagnostic Devices. We prepared two prototypes of diagnostic devices to illustrate some of the advantages of fully enclosed microPADs (Figure 4). The first prototype contains a sample inlet hole and holes over the test zones in the top layer of toner (Figure 4A-D). The inlet hole allows the user to add the sample to the device using a pipet or dropper. A drop of blood from a finger prick could also be added directly to the sample inlet. Since the back of the device is sealed with toner, the device can be placed on a table or other surface when it is in use. The holes over test zones allow visualization of the results.
The second prototype is fully enclosed (Figure $4 \mathrm{E}-\mathrm{H}$ ). It has no holes in the toner and would not require any additional packaging. To operate the device, the user cuts the bottom portion of the device off and then dips the bottom of the device into the sample. A sample inlet at the bottom of the device wicks the sample into the device and through the reagentstorage zones, where it is combined with reagents. The sample and reagents are then wicked into the test zones, where the results are displayed. The test zones were sealed with yellow toner so that the results could be visualized. Comparing the results in the test zones from the two devices in Figure 4 shows that the layer of yellow toner negatively impacts the ability to visualize the results. Laser printers that print clear toner, such as the Canon imagePRESS $\mathrm{C} 1+$, are commercially available, and we envision using this type of printer to cover the test zones with clear toner so that they could be enclosed without obscuring the results.

Both prototype devices have instructions printed on them so that an inexperienced user could perform the assays without any training. Additional instructions, labels, or barcodes could be printed on the back of the device. The devices also include an area for labeling. This area was covered with yellow wax during the patterning process so that it would be hydrophobic, but it is still possible to write in this area using a pen or pencil. The labeled device serves as physical evidence of the assay and can be stored as part of a patient's medical records.

\section{CONCLUSIONS}

Fully enclosed microPADs fabricated by laser printing represent a new class of paper-based microfluidic devices with many advantages over conventional open-channel microPADs. Enclosing microPADs protects the channels and reagents from contamination, increases the wicking rate of fluids in the channels, and may eliminate the need for individual packaging, which could significantly reduce the final cost of a commercial microPAD. A final advantage of this method for enclosing devices is that instructions, labels, and barcodes can be added to microPADs without requiring any additional fabrication steps. Fully enclosed microPADs should be compatible with all of the other technologies currently being developed for microPADs, ${ }^{18-22}$ and the fabrication technique will bring new capabilities to this class of analytical devices.

\section{ASSOCIATED CONTENT}

\section{S Supporting Information}

Additional information as noted in text. This material is available free of charge via the Internet at http://pubs.acs.org.

\section{AUTHOR INFORMATION}

\section{Corresponding Author}

*E-mail: awmartin@calpoly.edu. Phone: 805-756-2744.

\section{ACKNOWLEDGMENTS}

This project was supported by the California State University Program for Education and Research in Biotechnology (CSUPERB). We thank Chad Immoos and Derek Gragson for helpful discussions and assistance with the microscope cameras.

\section{REFERENCES}

(1) Martinez, A. W.; Phillips, S. T.; Butte, M. J.; Whitesides, G. M. Angew. Chem., Int. Ed. 2007, 46, 1318-1320. 
(2) Martinez, A. W.; Phillips, S. T.; Whitesides, G. M.; Carrilho, E. Anal. Chem. 2010, 82, 3-10.

(3) Fenton, E. M.; Mascareñas, M. R.; Lopez, G. P.; Sibbett, S. S.

ACS Appl. Mater. Interfaces 2009, 1, 124-129.

(4) Martinez, A. W.; Phillips, S. T.; Whitesides, G. M. Proc. Natl. Acad. Sci. U.S.A. 2008, 105, 19606-19611.

(5) Olkkonen, J.; Lehtinen, K.; Erho, T. Anal. Chem. 2010, 82, 10246-10250.

(6) Fu, E.; Lutz, B.; Kauffman, P.; Yager, P. Lab Chip 2010, 10, 918920.

(7) Coltro, W. K.; de Jesus, D. P.; da Silva, J. A.; do Lago, C. L.; Carrilho, E. Electrophoresis 2010, 15, 2487-2498.

(8) Bracher, P. J.; Gupta, M.; Mack, E. T.; Whitesides, G. M. ACS Appl. Mater. Interfaces 2009, 8, 1807-1812.

(9) Bracher, P. J.; Gupta, M.; Whitesides, G. M. Soft Matter 2010, 6, 4303-4309.

(10) Samsung CLP-620 Series User Manual; Samsung Electronics Co. Ltd.: Seoul, South Korea, 2009.

(11) Martinez, A. W.; Phillips, S. T.; Carrilho, E.; Thomas, S. W.; Sindi, H.; Whitesides, G. M. Anal. Chem. 2008, 80, 3699-3707.

(12) Carrilho, E.; Martinez, A. W.; Whitesides, G. M. Anal. Chem. 2009, 81, 7091-7095.

(13) Klasner, S. A.; Price, A. K.; Hoeman, K. W.; Wilson, R. S.; Bell,

K. J.; Culberston, C. T. Anal. Bioanal. Chem. 2010, 397, 1821-1829.

(14) Li, X.; Tian, J.; Shen, W. Cellulose 2010, 17, 649-659.

(15) Schechner, Z.; Luo, G.; Kaufman, J. J.; Siffert, R. S. Med. Biol. Eng. Comput. 2010, 48, 799-810.

(16) Evans, M. A.; LeMarie, B. A. An Investigation into Papers for Digital Printing; Printing Industry Center at RIT: Rochester, NY, 2006.

(17) Washburn, E. W. Phys. Rev. 1921, 17, 273-283.

(18) Delaney, J. L.; Hogan, C. F.; Tian, J.; Shen, W. Anal. Chem. 2011, 83, 1300-1306.

(19) Fu, E.; Liang, T.; Houghtaling, J.; Ramachandran, S.; Ramsey, S. A.; Lutz, B.; Yager, P. Anal. Chem. 2011, 83, 7941-7946.

(20) Noh, H.; Phillips, S. T. Anal. Chem. 2010, 82, 8071-8078.

(21) Nie, Z.; Nijhuis, C. A.; Gong, J.; Chen, X.; Kumachev, A.; Martinez, A. W.; Narovlyansky, M.; Whitesides, G. M. Lab Chip 2010, 10, 477-483.

(22) Carrilho, E.; Phillips, S. T.; Vella, S. J.; Martinez, A. W.; Whitesides, G. M. Anal. Chem. 2009, 81, 5990-5998. 